\title{
Treatment of intrabony defects with GTR increases clinical attachment and bone
}

\author{
Cortellini P, Carnevale G, Sanz M, Tonetti MS. Treatment of deep and shallow intrabony defects. A multicenter \\ randomized controlled clinical trial. J Clin Periodontol 1998; 25:981-987
}

Objective To compare the efficacy of guided tissue regeneration (GTR) in intra-bony defects with bioresorbable barrier membranes versus access flap surgery.

Design A prospective multi-centre randomised controlled trial in three periodontal practices. Each patient received both the control and test treatment.

Intervention Two similar defects were selected in each of 23 patients and randomly assigned to one of the two treatments. Surgery consisted of an identical procedure except for the omission of the barrier membrane in the flap control sites.

Outcome measures Full mouth plaque scores (FMPS), Bleeding on probing (using Florida probe), Full mouth bleeding scores (FMBS), Pocket probing depth (PPD) and clinical attachment level (CAL) were recorded before surgery, and after 6 months and 1 year. Probing pocket depth reduction and clinical attachment level gains were evaluated.

Results Use of the bioresorbable membrane resulted in mean pocket depths that were significantly less $(\geqslant 1.3 \mathrm{~mm}, p=0.02)$ and mean clinical attachments that were significantly greater $(\geqslant 1.4 \mathrm{~mm}$, $p<0.01)$ than controls. Three intrabony sites would need to be treated with flap surgery and a bioresorbable membrane for one site to demonstrate an attachment level gain of $<2 \mathrm{~mm}$.

Conclusions The present study demonstrated that GTR with bioresorbable barrier membranes resulted in a significant added benefit in comparison with access flap alone. The linear amounts of CAL gains were greater in deep than in shallow defects.

Address for reprints: Pierpaolo Cortellini, Via Carlo Botta 16, 50136 Firenze, Italy.

\section{Commentary}

Guided tissue regeneration techniques are part of advanced periodontics but their success is dependent on many factors. These include patient characteristics, variations in defect morphology, design of the surgical technique, the type of barrier membrane, experience of the operator and post-treatment plaque control and supportive care. This well-designed study has clearly set out inclusion criteria, near identical defects in the same jaw subjected to surgery at the same session and meticulous after care and follow-up.

A number of issues, however, arise when considering the results of this study. The age range of the subjects is considerable but data are not stratified according to age groups, probably because of the relatively small numbers of subjects. Does age play a role in regeneration? This is important clinically as, in routine practice, GTR procedures are perhaps more likely to be carried out in younger patients.

The study evaluates outcomes over a one-year period, during which meticulous professional support for plaque control is provided. Results over longer periods (with normal periodontal supportive care) are needed to assess the effectiveness of the procedure. Indeed, it is the very variations identified at the beginning which confront the clinicians in daily practice and require careful analysis in different settings to truly establish the effectiveness of GTR.

The stratification of the data into shallow and deep intra-bony defects demonstrates the overall regeneration potential in the infra-bony periodontal defects. However, furcation involvement was excluded from the trial and it is often such complex defects which require intensive treatment. Furthermore, the tooth types are also not identified. The application of the results to clinical practice is thus limited.

Periodontal studies usually use disease-orientated outcomes, but it is increasingly obvious that patientorientated outcomes are more important to patients! Thus, such factors as post-operative pain, inconvenience of intensive regular professional prophylaxis, tooth loss, sensitivity and costs should also be evaluated so that effectiveness and cost-effectiveness can be assessed and the utility of the treatments to the patient made explicit.

This study establishes the efficacy of using a polylactic acid barrier membrane but further research is necessary to establish its effectiveness and its relationship with other types of membranes used in GTR.

\section{Rajenedra I Joshi}

Charles Clifford Dental Hospital, Sheffield, UK 